\title{
Intraocular Invasion of Ocular Surface Squamous Neoplasia Through a Corneal Wound
}

Diana M. Laura - Anastasia Gkiala - Georgios Charonis •

Sotiria Palioura

Received: July 21, 2020 / Published online: August 31, 2020

(C) The Author(s) 2020

\section{ABSTRACT}

Purpose: We report a case of intraocular invasion of ocular surface squamous neoplasia (OSSN) through a cataract surgery wound that manifested as an anterior chamber membrane, and perform a review of the literature.

Methods: An 87-year-old woman with history of an incompletely excised OSSN 10 days prior presented with decreased vision due to an anterior chamber membranous film that seemed connected to an old cataract wound. The membrane was biopsied, and histopathology revealed dysplastic squamous epithelial cells of conjunctival origin. Ten days later the

Digital Features To view digital features for this article go to https://doi.org/10.6084/m9.figshare.12794459.

Electronic supplementary material The online version of this article (https://doi.org/10.1007/s40123020-00294-2) contains supplementary material, which is available to authorized users.

D. M. Laura $\cdot$ S. Palioura $(\bowtie)$

Department of Ophthalmology, Bascom Palmer Eye Institute, University of Miami Miller School of

Medicine, Miami, FL, USA

e-mail: sotiria.palioura@gmail.com

A. Gkiala

National and Kapodistrian University of Athens

School of Medicine, Athens, Greece

G. Charonis · S. Palioura

Athens Vision Eye Institute, Athens, Greece tumor expanded to cover the entire iris surface, and a modified enucleation was performed.

Results: Histopathology revealed invasive OSSN extending deep to the ciliary body and laterally into the anterior chamber. The patient developed a submandibular node metastasis 7 months later. Literature review revealed six cases of invasive OSSN resulting from extension through a corneal wound, with varied presentations and outcomes.

Conclusion: Our case is the first to report invasive OSSN presenting as an anterior chamber membrane as a result of incomplete excision of an ocular surface malignancy adjacent to a corneal wound poses a risk for intraocular tumor extension and distant metastasis.

Keywords: Anterior chamber membrane; Cataract wound; Conjunctival squamous cell carcinoma; Intraocular extension; OSSN 


\section{Key Summary Points}

This report describes a case of intraocular invasion of an ocular surface squamous neoplasia (OSSN) through a prior cataract wound.

Unlike the six prior reported cases, wherein the invasive OSSN lesion presented as a mass or corneal stromal opacity, in this case it formed a dense membrane in the anterior chamber.

Our report, along with the few prior cases in the literature, demonstrates that patients with tumor cells on the ocular surface may be at increased risk of invasive disease either at the time of cataract surgery or years after it, if the malignancy is not diagnosed and/or managed properly.

In a patient with current or prior suspected surface malignancy and history of incisional surgery presenting with intraocular inflammation, membranes, or masses, intraocular OSSN must be considered.

\section{DIGITAL FEATURES}

This article is published with digital features to facilitate understanding of the article. You can access the digital features on the article's associated Figshare page. To view digital features for this article go to https://doi.org/10.6084/m9. figshare.12794459.

\section{INTRODUCTION}

Ocular surface squamous neoplasia (OSSN) refers to a slowly progressive malignant lesion of the conjunctiva and cornea and encompasses squamous dysplasia, conjunctival intraepithelial neoplasia and in situ or invasive squamous cell carcinoma [1]. These neoplasms typically occur in the mitotically active limbal region within the interpalpebral zone. Risk factors for OSSN include ultraviolet light exposure, human papilloma virus and human immunodeficiency virus infection, cigarette smoking, ocular surface injury and exposure to chemicals $[1,2]$. Surgical excision with wide surgical margins, a no-touch technique and intraoperative cryotherapy is considered the gold standard in the management of OSSN [2]. Alternative recommended treatments that have gained popularity in recent years include topical chemotherapy agents such as mitomycin-C (MMC), 5-fluorouracil and interferon alpha-2b [2].

The clinical course of OSSN is typically that of a localized and slowly growing lesion. Rarely OSSN has been described to extend intraocularly through the sclera, with vision-, eye- and life-threatening consequences [3, 4]. Risk factors for scleral invasion include mucoepidermoid, spindle cell and poorly differentiated variants of carcinoma, as well as history of immunosuppression and infection with the human immunodeficiency virus [3, 4].

There are very few cases in the literature describing intraocular OSSN tumor extension through a corneal wound, with clinical presentations ranging from intrastromal corneal opacities to anterior chamber masses [5-9]. Herein, we report a case of intraocular OSSN invasion through a prior cataract surgery incision that presented as a rapidly growing anterior chamber membrane, with treatment requiring modified enucleation with a dermis fat graft. The study was conducted according to the tenets of the Declaration of Helsinki. The patient signed an informed consent form for publication of the case.

\section{CASE PRESENTATION}

An 87-year-old woman with history of rheumatoid arthritis presented with pain, decreased vision and injection in her right eye 10 days after excisional biopsy of a conjunctival lesion and placement of a conjunctival autograft. Per records, cryotherapy or antimetabolites were not used intraoperatively. The lesion 
A

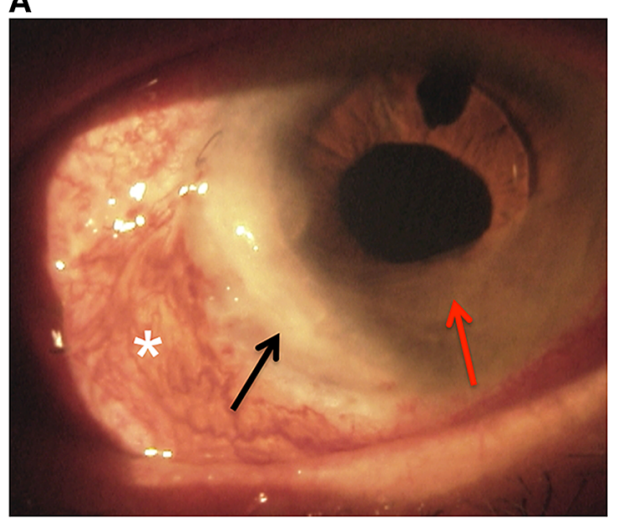

B

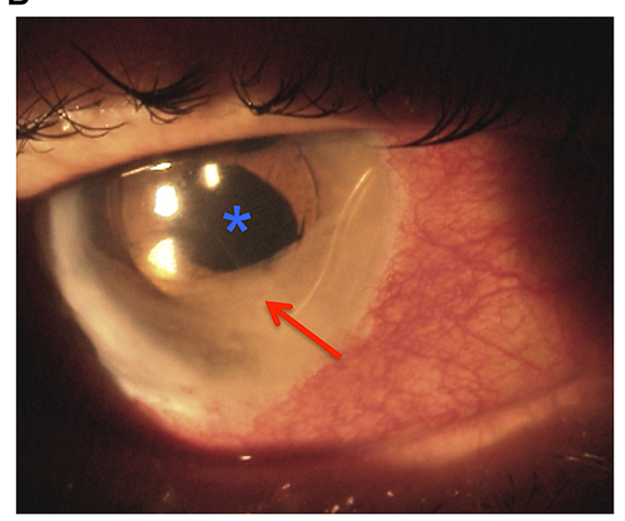

C

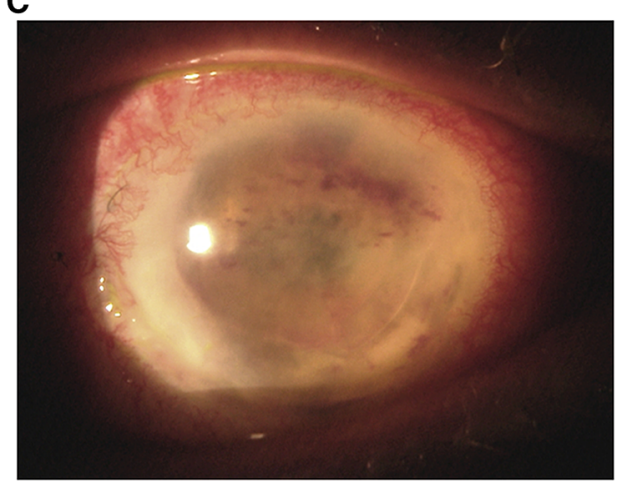

Fig. 1 Slit lamp photograph illustrating a membranous film in the anterior chamber of the patient's right eye at presentation (a, b) and 10 days later (c). A conjunctival autograft is in place temporally (white asterisk). The anterior chamber membrane (red arrow) is visualized extending from a cataract incision (black arrow) and covering the anterior surface of the ACIOL (blue asterisk)

was adjacent to a 1-year old cataract wound. Histopathology revealed poorly differentiated OSSN with positive surgical margins.
On initial exam, Snellen visual acuity in the right eye was 20/320. A membranous film covering the inferior half of an anterior chamber intraocular lens (ACIOL) and extending to the old cataract incision was seen on slit lamp examination of the right eye (Fig. 1a, b). The membrane was not attached to the posterior corneal surface. The membrane was biopsied in the operating room. Two paracentesis incisions were made superiorly, the membrane was grasped with micro forceps (MST, Redmond, WA), a small sample was cut with micro scissors (MST), and it was fixed in formalin for histopathological evaluation. Histopathology revealed dysplastic squamous epithelial cells of conjunctival origin. The patient and her family declined enucleation at that time given her age and comorbidities.

Ten days after initial presentation, her vision declined to light perception, and the membrane expanded to cover the entirety of the ACIOL and iris surface (Fig. 1c). Given the rapid progression, the patient was then amenable to surgery. A modified enucleation was performed using a no touch technique with wide margins and cryotherapy. A dermis fat graft was used instead of an implant given that a significant area of the conjunctiva was sacrificed to achieve clear margins, and the patient would be at risk for implant exposure. Histopathology revealed invasive squamous cell carcinoma extending deep to the ciliary body and laterally into the anterior chamber, with negative conjunctival margins. No mucoepidermoid or spindle cell characteristics were observed. The patient developed a submandibular node metastasis 7 months after enucleation.

\section{DISCUSSION}

This report presents a rare case of intraocular OSSN invasion through a prior cataract surgery wound that manifested as a rapidly expanding anterior chamber membranous structure shortly after incomplete excision of a conjunctival OSSN lesion adjacent to the cataract incision. Despite modified enucleation with clean conjunctival margins, the patient developed a regional metastasis 7 months later. 


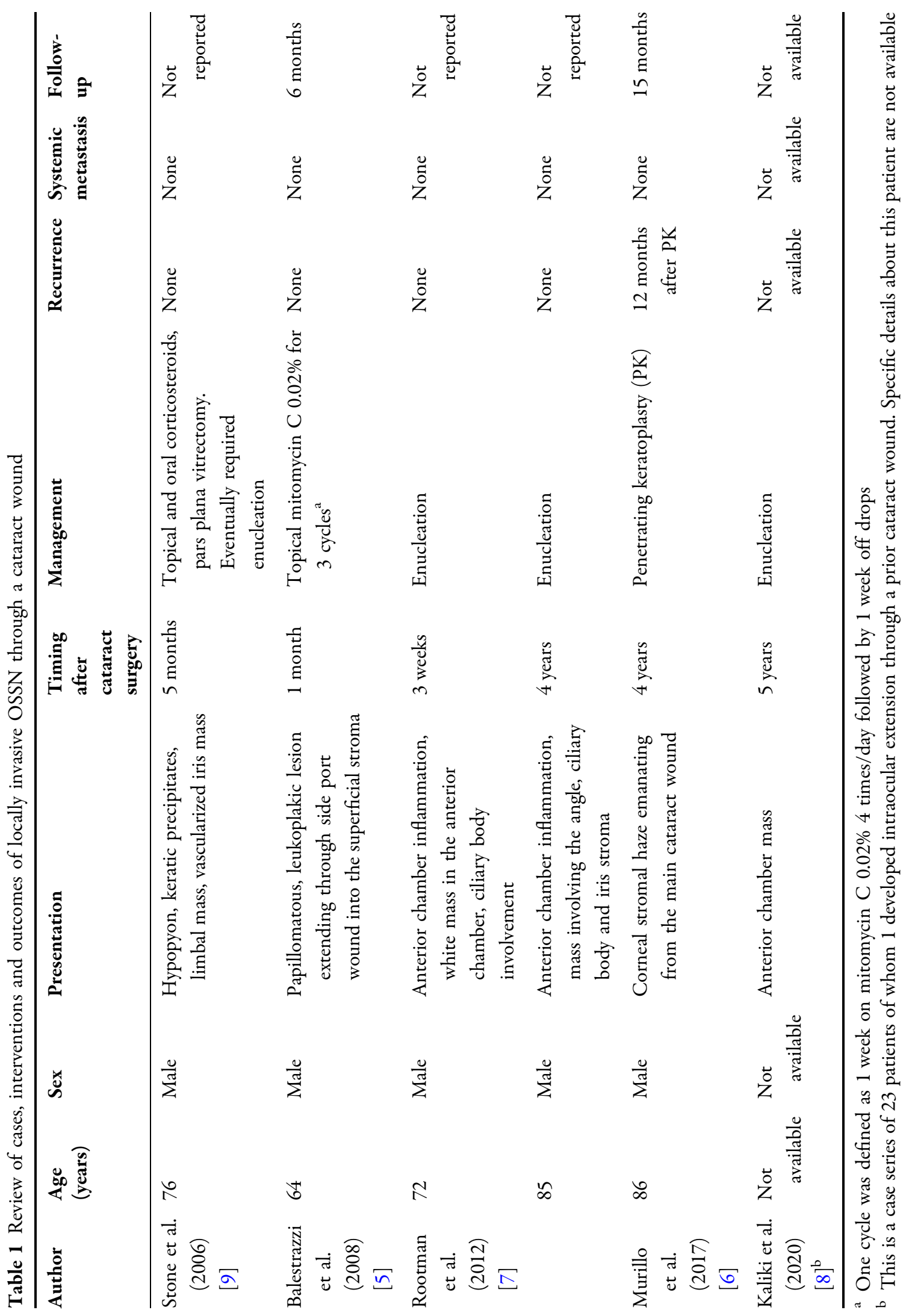


Intraocular extension of OSSN is a rare entity, reported in $1-15 \%$ of cases $[8,10]$. Tumor extension can occur either through direct scleral invasion, invasion along the tract of the anterior ciliary vessels, or tumor inoculation through an intraocular surgery incision. A literature review revealed six reports of invasive OSSN specifically resulting from extension through a corneal wound (Table 1) [5-9].

Two cases describe intraocular extension limited to corneal stromal invasion [5, 6]. In the first report, a patient with a presumed nasal pinguecula underwent uncomplicated cataract surgery [5]. One month after surgery, the patient was found to have a papillomatous, leukoplakic lesion extending nasally through the side port wound into the superficial stroma [5]. The patient was treated with three cycles of MMC with resolution of the lesion and no recurrence for 6 months [5]. In the second report, a patient with no noted prior conjunctival lesions underwent uncomplicated cataract surgery, and 3 months later was found to have biopsy-confirmed OSSN near the cataract wound, which was excised with cryotherapy, MMC and amniotic membrane reconstruction [6]. He then developed recurrences 1 and 2 years later, which were treated successfully with topical chemotherapy [6]. However, 4 years after surgery, corneal haze was seen extending from the cataract wound, a penetrating keratoplasty was performed, and histopathology revealed mid-stromal infiltrative carcinoma [6]. The patient subsequently experienced recurrence, but ultimately died of other health issues.

In the other four reports of OSSN extension through a cataract incision, a more aggressive course was noted, with the appearance of iris and angle masses [7-9]. All four patients required enucleation, but none developed a metastatic lesion [7-9]. One case described the development of a contiguous vascular mass extending from the limbus at the surgical incision site to the iris [9]. This lesion was preceded by chronic inflammation that was intractable to prednisone and pars plana vitrectomy [9]. The eye subsequently became pre-phthisical and required enucleation [9]. In another report, two cases of incompletely excised conjunctival OSSN adjacent to a cataract wound resulted in intraocular inflammation, development of an intraocular mass involving the angle, ciliary body and iris stroma, and subsequently underwent enucleation [7].

Surgical intervention poses a risk for intraocular OSSN extension by creating a tract for tumor cells to enter the eye. It has been postulated that this is likely due to intraoperative disruption of the integrity of Bowman's layer, which is thought to be tumor-protective $[1,6]$. Based on the reviewed reports, the presence of an OSSN lesion at the time of cataract surgery led to incisional invasion of the tumor in the early postoperative period ( 3 weeks- 5 months postoperatively) in three cases $[5,7,9]$. In the remaining cases, tumor invasion occurred $4-5$ years after cataract surgery due to incomplete excision of an OSSN lesion adjacent to an old cataract wound [6-8]. Therefore, timely diagnosis and appropriate management of OSSN both prior to and after cataract surgery is of utmost importance to prevent intraocular tumor extension and its catastrophic consequences.

\section{CONCLUSION}

In conclusion, our report, along with the few prior cases in the literature, demonstrates that patients with tumor cells on the ocular surface may be at increased risk of invasive disease either at the time of cataract surgery or years after it, if the malignancy is not diagnosed and/ or managed properly. Prior reported cases demonstrate that the clinical presentation of invasive intraocular OSSN in patients with a history of cataract extraction varies, ranging from stromal opacification to intraocular mass. Our report is the only case in the literature that describes a rapidly progressive anterior chamber membrane formation. Thus, in a patient with current or prior suspected surface malignancy and history of incisional surgery presenting with intraocular inflammation, membranes, or masses, intraocular OSSN must be considered. 


\section{ACKNOWLEDGEMENTS}

We would like to thank our patient for giving her consent for the publication of this case.

Funding. No funding or sponsorship was received for this study or publication of this article. The Rapid Service Fee was funded by the authors.

Authorship. All named authors meet the International Committee of Medical Journal Editors (ICMJE) criteria for authorship for this article, take responsibility for the integrity of the work as a whole, and have given their approval for this version to be published.

Disclosures. Sotiria Palioura is a consultant for Alcon. Diana M. Laura, Anastasia Gkiala and Georgios Charonis have nothing to disclose.

Compliance with Ethics Guidelines. The study was conducted according to the tenets of the Declaration of Helsinki. The patient signed an informed consent form for publication of the case.

Data Availability. Data sharing is not applicable for this article, as no data sets were generated or analyzed.

Open Access. This article is licensed under a Creative Commons Attribution-NonCommercial 4.0 International License, which permits any non-commercial use, sharing, adaptation, distribution and reproduction in any medium or format, as long as you give appropriate credit to the original author(s) and the source, provide a link to the Creative Commons licence, and indicate if changes were made. The images or other third party material in this article are included in the article's Creative Commons licence, unless indicated otherwise in a credit line to the material. If material is not included in the article's Creative Commons licence and your intended use is not permitted by statutory regulation or exceeds the permitted use, you will need to obtain permission directly from the copyright holder. To view a copy of this licence, visit http://creativecommons.org/licenses/by$\mathrm{nc} / 4.0 /$.

\section{REFERENCES}

1. Lee GA, Hirst LW. Ocular surface squamous neoplasia. Surv Ophthalmol. 1995;39(6):429-50.

2. Sayed-Ahmed IO, Palioura S, Galor A, et al. Diagnosis and medical management of ocular surface squamous neoplasia. Expert Rev Ophthalmol. 2017;12(1):11-9.

3. Shields JA, Shields CL, Gunduz K, et al. The 1998 Pan American Lecture. Intraocular invasion of conjunctival squamous cell carcinoma in five patients. Ophthalmic Plast Reconstr Surg. 1999;15(3):153-60.

4. Chauhan S, Sen S, Sharma A, et al. American Joint Committee on Cancer Staging and clinicopathological high-risk predictors of ocular surface squamous neoplasia: a study from a tertiary eye center in India. Arch Pathol Lab Med. 2014;138(11): 1488-94.

5. Balestrazzi A, Martone G, Pichierri P, et al. Corneal invasion of ocular surface squamous neoplasia after clear corneal phacoemulsification: in vivo confocal microscopy analysis. J Cataract Refract Surg. 2008;34(6):1038-43.

6. Murillo JC, Galor A, Wu MC, et al. Intracorneal and intraocular invasion of ocular surface squamous neoplasia after intraocular surgery: report of two cases and review of the literature. Ocul Oncol Pathol. 2017;3(1):66-72.

7. Rootman DB, McGowan HD, Yucel YH, et al. Intraocular extension of conjunctival invasive squamous cell carcinoma after pterygium surgery and cataract extraction. Eye Contact Lens. 2012;38(2):133-6.

8. Kaliki S, Jajapuram SD, Maniar A, et al. Ocular surface squamous neoplasia with intraocular tumour extension: a study of 23 patients. Eye. 2020;34(2): 319-26.

9. Stone DU, Char DH, Crawford JB, et al. Metaplastic squamous epithelial downgrowth after clear corneal cataract surgery. Am J Ophthalmol. 2006;142(4): $695-7$.

10. Yousef YA, Finger PT. Squamous carcinoma and dysplasia of the conjunctiva and cornea: an analysis of 101 cases. Ophthalmology. 2012;119(2):233-40. 